\title{
ÖGUM-Vorstand 2017-2020
}

Bei der Generalversammlung der ÖGUM anlässlich des Dreiländertreffens in Linz wurde der Vorstand neu gewählt. Im Namen des neuen Vorstandes bedanke ich mich bei allen, die uns gewählt und mit einem Vertrauensvorschuss ausgestattet haben. Ich bedanke mich auch bei unseren KollegInnen aus dem alten Vorstand sehr herzlich für die ehrenamtliche Arbeit und ihr Engagement. Es soll nicht unerwähnt bleiben, dass alle Tätigkeiten für die Gesellschaft neben der belastenden Routine in Spitälern und Praxen erfolgen. Wir übernehmen eine von Frau Univ. Prof. Andrea Klauser umsichtig geführte, wohl geordnete und gut aufgestellte Gesellschaft. Frau Univ. Prof. Andrea Klauser steht als PastPräsidentin nicht mehr zur Verfügung, was wir sehr bedauern. Sie wird uns aber mit guten Ratschlägen und Informationen weiter unterstützen. Das gleiche gilt für den früheren Präsidenten Univ. Prof. Dr. Horst Steiner, für PD Dr. Helmut Prosch und Ass. Prof. Dr. Christian Kollmann. Letztere sind aus beruflichen Gründen ausgeschieden. President elect ist Frau Univ. Prof. Dr. Barbara Pertl (Graz), PD Dr. Hannes Gruber (Innsbruck) übernahm die Agenden des Sekretärs, PD Dr. Stefan Meng die des Kassiers, Frau Dr. Doris Kerö (St. Pölten) bleibt als weiteres Mitglied im Vorstand. Neu im Vorstand sind Prim. PD Dr. Wolfgang Arzt, der den Linzer Kongress perfekt organisiert hat und Dr. Peter Michael Zechner (Graz), der als Kardiologe/Internist und Notfallmediziner einen breiten Bereich abdeckt. Wir hoffen, dass so im Vorstand ein ausgewogenes Verhältnis zwischen den Vertreterlnnen der Universitäten, der Fachrichtungen und der niedergelassene Kollegenschaft entstanden ist.

Unser erstes Ziel soll sein, den guten Zustand der Gesellschaft, das Arbeitsklima und den Zusammenhalt so zu erhalten. Wir sind, bedingt durch die demografische
Entwicklung, eine alternde Gesellschaft und die Mitgliederzahlen stagnieren. Wir müssen daher versuchen, unsere Mitgliederzahl zu erhöhen und junge KollegInnen in die Gesellschaft zu bringen. Das ÖGUMKurswesen, die Dreiländertreffen und die für Mitglieder günstige Zeitschrift „Ultraschall in der Medizin" bieten in diesem Zusammenhang ein attraktives Angebot. Eine weitere Chance liegt darin, die Aktivitäten von Studierenden zu unterstützen und sie sukzessive als aktive Mitglieder in die ÖGUM einzuarbeiten. Die an einigen Universitäten des Landes existierenden „Sono4You“ - Gruppen begeistern die interessierten StudentInnen als „Auffangorganisation“ für den Ultraschall und münden seit 2016 in einen eigens durch die ÖGUM geschaffener Arbeitskreis mit ausgesprochen attraktiven Angeboten an die motivierten studentischen ÖGUM-Mitglieder. Wir sehen es als dringliche Aufgabe der ÖGUM an, die Studierenden bei ihren Tätigkeiten strukturiert zu unterstützen. Dazu gehört, dass mit den Universitäten kommuniziert wird, dass die ÖGUM diese freiwilligen Aktivitäten von Studierenden unterstützt und wertschätzt.

Fachspezifische Ausbildung in Ultraschall ist in vielen aktualisierten Ausbildungsordnungen verankert. Die Umsetzung ist aber aus verschiedenen Gründen nicht immer einfach. Das betrifft sowohl die Erbringung der geforderten Fallzahlen, als auch die kontinuierliche Ausbildung am Patienten unter Aufsicht erfahrener KollegInnen. Realistisch gesehen wird die ÖGUM nur bedingt Einflüsse auf innerbetriebliche, klinische Abläufe haben können, sieht sich jedoch in der Verantwortung, weiter auf die Notwendigkeit einer suffizienten ärztlichen Ausbildung hinzuweisen.

Ich bin überzeugt, dass die Mitglieder der ÖGUM den Schallkopf als modernes Stetho-

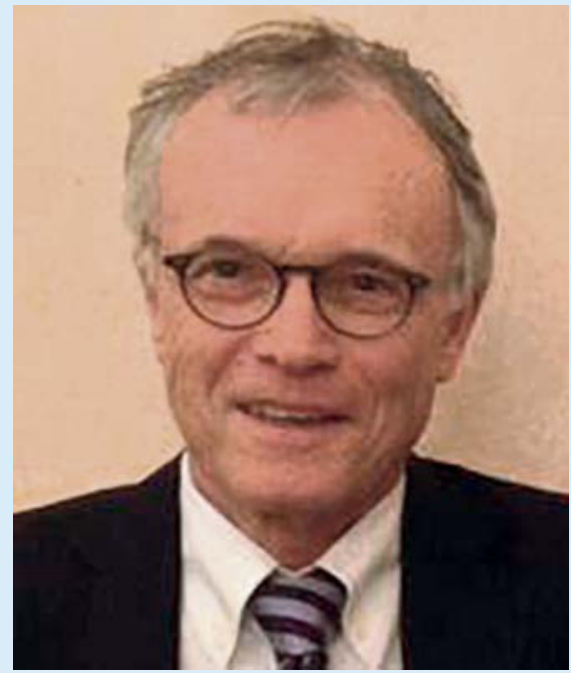

ÖGUM-Präsident Univ. Prof. Dr. Hermann Kathrein

skop, und Ultraschall als wesentlichen Teil der Diagnostik sehen. Das Arbeiten mit dem Schallkopf in der erwähnten Weise, d. h. als „Scheinwerfer“ der spezifisch ärztlichen Diagnostik, ist ja auch Tradition gerade der deutschsprachigen Ländergesellschaften. Die ÖGUM lehnt es daher ab, die Arbeit am Patienten mit dem Ultraschallgerät an nicht-ärztliche Personengruppen zu delegieren. Diese Thematik wird uns aber aufgrund sich ändernder Organisationsstrukturen weiter beschäftigen. Die Diskussion darüber muss sorgsam und verantwortungsvoll geführt werden.

Weiters müssen wir uns, das haben Anfragen in der Vollversammlung gezeigt, wieder um die Harmonisierung von Kursen kümmern. Derzeit sieht es so aus, dass lediglich für den Bereich Abdomen die gegenseitige Anerkennung gut verankert ist. Dieses Thema wird beim nächsten Treffen der Vorstände im April 2018 zu besprechen sein. Ich halte eine schnelle einfache Lösung, trotz ernsthafter Bemühungen aller, aber für nicht realistisch. 
Im letzten Jahr sind mehrere Kollegen aus der ÖGUM mit ehrenvolle Aufgaben bei der Zeitschrift „Ultraschall in der Medizin“ und in der Europäischen Gesellschaft (ESUMB) betraut worden: PD. Dr. S. Meng ist in das Editorial Board der Zeitschrift aufgenommen worden. Univ. Prof. G. Mathis wurde zum EFSUMB Fellow erwählt, PD Dr. $\mathrm{H}$. Prosch zum Member im Education and Professional Standards Committee (EPSC), Ass. Prof. Dr. C. Kollmann zum Chairman im Safety Committee (ECMUS), und Herr cand.med. Philipp Niederkofler zum Mit- glied im EFSUMB Student Committee. Das zeigt, wie sehr die Arbeit von ÖGUM-Mitgliedern, aber auch von Studierenden anerkannt wird. Andererseits sehen viele von uns- das ist zumindest mein Eindruck - die europäische Gesellschaft als eher ferne Institution. Wir sollten überlegen, wie wir Angebote und Möglichkeiten der EFSUMB besser nützen können. Ich denke da vor allem an Möglichkeiten für die Studierenden (und hoffentlich zukünftigen ÖGUM-Mitglieder).
Zum Abschluss möchte ich Ihnen versichern, dass wir alle im Vorstand für Ihre Anliegen offen sind und uns bemühen werden, Ihnen nach Möglichkeit bestens zu helfen. Schreiben Sie uns, wenn Sie etwas brauchen. Last but not least bedanke ich mich im Namen aller bei Frau Beate Kasperak und Frau Verena Schoiswohl, die im ÖGUM Sekretariat exzellent Arbeit machen.

Für den ÖGUM Vorstand

Univ. Prof. Dr. Hermann Kathrein ÖGUM Präsident 